Check for updates

Cite this: RSC Adv., 2018, 8, 29855

Received 23rd July 2018

Accepted 20th August 2018

DOI: $10.1039 / \mathrm{c} 8 \mathrm{ra06235h}$

rsc.li/rsc-advances

\section{Red C-dots and C-dot films: solvothermal synthesis, excitation-independent emission and solid-state-lighting ${ }_{\dagger}^{\dagger}$}

\begin{abstract}
Daqin Chen, (D) *ab Xiao Chen, ${ }^{a}$ Haobo Gao and Jiasong Zhong ${ }^{a}$
Currently, fluorescent carbon dots (CDs) have attracted great attention for their unique optical performance. However, the shortage of red emissive CDs and the corresponding CD solid samples with intense luminescence significantly limit their applications in optoelectronic fields. In the present work, red fluorescence CDs were successfully synthesized via a facile solvothermal reaction using $p$ phenylenediamine as the carbon source and isopropanol as the solvent. Excitation-independent luminescence and emission-independent decay indicated that one dominant type of emissive state was responsible for red luminescence, which was evidenced to be a N-related surface defect state with the help of structural and spectroscopic characterizations. Furthermore, CD-embedded PVA solid films, exhibiting bright red emission with intense absorption in the blue-light region, were prepared to explore their possible application as a color converter in solid-state lighting. As a proof-of-concept experiment, white light-emitting diode devices were constructed by combining a red CD solid film and yellow Ce:YAG phosphor-in-glass with a commercial InGaN blue chip, showing tunable color coordinates, color rendering index and correlated color temperature via modifying the thickness of the CD film.
\end{abstract}

\section{Introduction}

Because of their superiority in terms of excellent water solubility, optical stability, unique fluorescence performance, low toxicity and low cost, fluorescent carbon quantum dots, as a new type of nanomaterial, have been studied intensively over the past decade for potential applications in fluorescent probes, light emitting devices and biosensors. ${ }^{1-5}$ Since carbon dots (CDs) were discovered, various synthetic methods have been developed, and they can be largely divided into "top-down" and "bottom-up" routes. ${ }^{6-8}$ This classification is mainly based on carbon sources. The "top-down" route physically breaks macroscopic carbon materials into smaller nanomaterials, while the "bottom-up" route chemically generates $\mathrm{C}$-dots through molecular selfassembly reactions from various small-molecule reagents. ${ }^{9-12}$ Recently, the bottom-up route has attracted great attention and been widely used to prepare CDs since the precursor sources are abundant and the reaction is easy to operate.

${ }^{a}$ College of Materials \& Environmental Engineering, Hangzhou Dianzi University, Hangzhou, 310018, P. R. China. E-mail: dqchen@hdu.edu.cn

${ }^{b}$ College of Physics and Energy, Fujian Normal University, Fuzhou, Fujian, 350117, P. R. China. E-mail: dqchen@fjnu.edu.cn

$\dagger$ Electronic supplementary information (ESI) available: Fig. S1-S3. More PL spectra, absorption spectra and decay curves of red CDs. See DOI: $10.1039 / \mathrm{c} 8 \mathrm{ra} 06235 \mathrm{~h}$
Presently, many fluorescent CDs have been prepared, but most of them show dominant emissions in the blue-green spectral region, which limits their practical applications in optoelectronic fields. ${ }^{13-17}$ Therefore, it is highly desirable to develop CDs with intense and stable red emission. Furthermore, the conversion from CD solution into carbon solid-state powder is urgently required. Unfortunately, the luminescence of $\mathrm{CD}$ powder is generally far weaker than that of the corresponding CD solution, probably attributing to aggregationinduced emission quenching. ${ }^{18-23} p$-Phenylenediamine ( $p$-PD) has been previously studied as a new carbon source for preparing red-emissive C-dots; however, significantly diverse emissive features were found when different kinds of solvents were adopted. ${ }^{24,25}$ As a result, the origination of red luminescence was actually confusing and not well understood.

In the present work, we developed a facile one-pot solvothermal reaction to synthesize bright excitationindependent red-emissive $\mathrm{N}$-doped CDs using $p$-PD as the precursor and isopropanol (IPA) as the solvent. Series of structural and optical spectroscopic characterizations were carried out to evidence that $\mathrm{N}$-related defect states on the surfaces of CDs were responsible for the red luminescence. Importantly, the obtained CDs can be easily converted into solid CD film with retained intense red luminescence. As a concept-of-experiment, the red-emissive $\mathrm{CD}$ film was used as color converter to demonstrate its promising application in solid-state-lighting. 


\section{Experimental section}

\section{Synthesis of red CDs}

$0.25 \mathrm{mmol}$ PPD was first dissolved in $20 \mathrm{~mL}$ isopropanol (IPA), and stirred to form a transparent solution. Then the solution was transferred into a $25 \mathrm{~mL}$ Teflon-lined stainless steel autoclave. After heating at $200{ }^{\circ} \mathrm{C}$ in an oven for $1 \mathrm{~h}$ and cooling to room temperate naturally, the resulted solution was centrifuged to remove large particles and red suspension was achieved. Finally, the solution was transferred into a vacuum freeze drier for drying 2 days to obtain C-dot solid powders.

\section{Preparation of CD films}

Polyvinyl alcohol (PVA, $2.0 \mathrm{~g}$ ) was firstly dissolved in $10 \mathrm{~mL}$ ultrapure water at $100{ }^{\circ} \mathrm{C}$ and then red C-dots IPA solution was dropwise added to produce C-dot aqueous solution (CD contents: $0.1-0.4 \mathrm{mg} \mathrm{mL} \mathrm{m}^{-1}$ ). After stirring for 5 minutes, the resulting mixture was transferred into a clean glass substrate and heated in a vacuum oven at $60{ }^{\circ} \mathrm{C}$ for $24 \mathrm{~h}$ to obtain a solid composite film.

\section{Preparation of $\mathrm{Ce}^{3+}: \mathrm{Y}_{3} \mathrm{Al}_{5} \mathrm{O}_{12}$ (Ce:YAG) phosphor-in-glass (PiG) plate}

A low-temperature co-sintering route was adopted to fabricate yellow-emissive Ce:YAG PiG plate. Low-melting glass with composition of $40 \mathrm{SnF}_{2}-30 \mathrm{SnO}-30 \mathrm{P}_{2} \mathrm{O}_{5}-10 \mathrm{CsF}$ (in mol\%) and 3wt\% commercial Ce:YAG phosphor (XinLi Illuminant Co. Ltd.) were thoroughly mixed and sintered in a corundum crucible at $400{ }^{\circ} \mathrm{C}$ for $20 \mathrm{~min}$ under an ambient atmosphere. The melt was quenched into a pre-heated steel mold and then naturally cooled to room temperature. The as-prepared PiG was elaborately polished and cut into $\varphi 10 \mathrm{~mm}$ plate with a thickness of $0.5 \mathrm{~mm}$.

\section{Construction of white light-emitting diode (wLED)}

LED devices were designed by directly coupling the as-prepared C-dot film/PiG color converters on the InGaN blue chip. To tune optoelectronic parameters, different thicknesses of C-dot film were adopted. Opaque silica gels were filled around the edges of device in order to avoid the leakage of blue light.

\section{Characterization}

The actual compositions of CD samples were determined by Xray photoelectron spectroscopy (XPS) using a VG Scientific ESCA Lab Mark II spectrometer equipped with two ultra-high-vacuum 6 chambers. All the binding energies were referenced to the $\mathrm{C} 1 \mathrm{~s}$ peak of the surface adventitious carbon at $284.6 \mathrm{eV}$. Fourier transform infrared (FTIR) spectra were recorded on a Nicolet 6700 FTIR spectrometer in the range $400-4000 \mathrm{~cm}^{-1}$ using the $\mathrm{KBr}$ pellet technique. Raman spectra were recorded on a microscopic confocal Raman spectrometer (Renishaw Trade Co., Ltd., England) under the excitation of a $785 \mathrm{~nm}$ laser. Microstructure observation was performed on a JEOL JEM-2010 transmission electron microscope (TEM) at $200 \mathrm{kV}$ accelerating
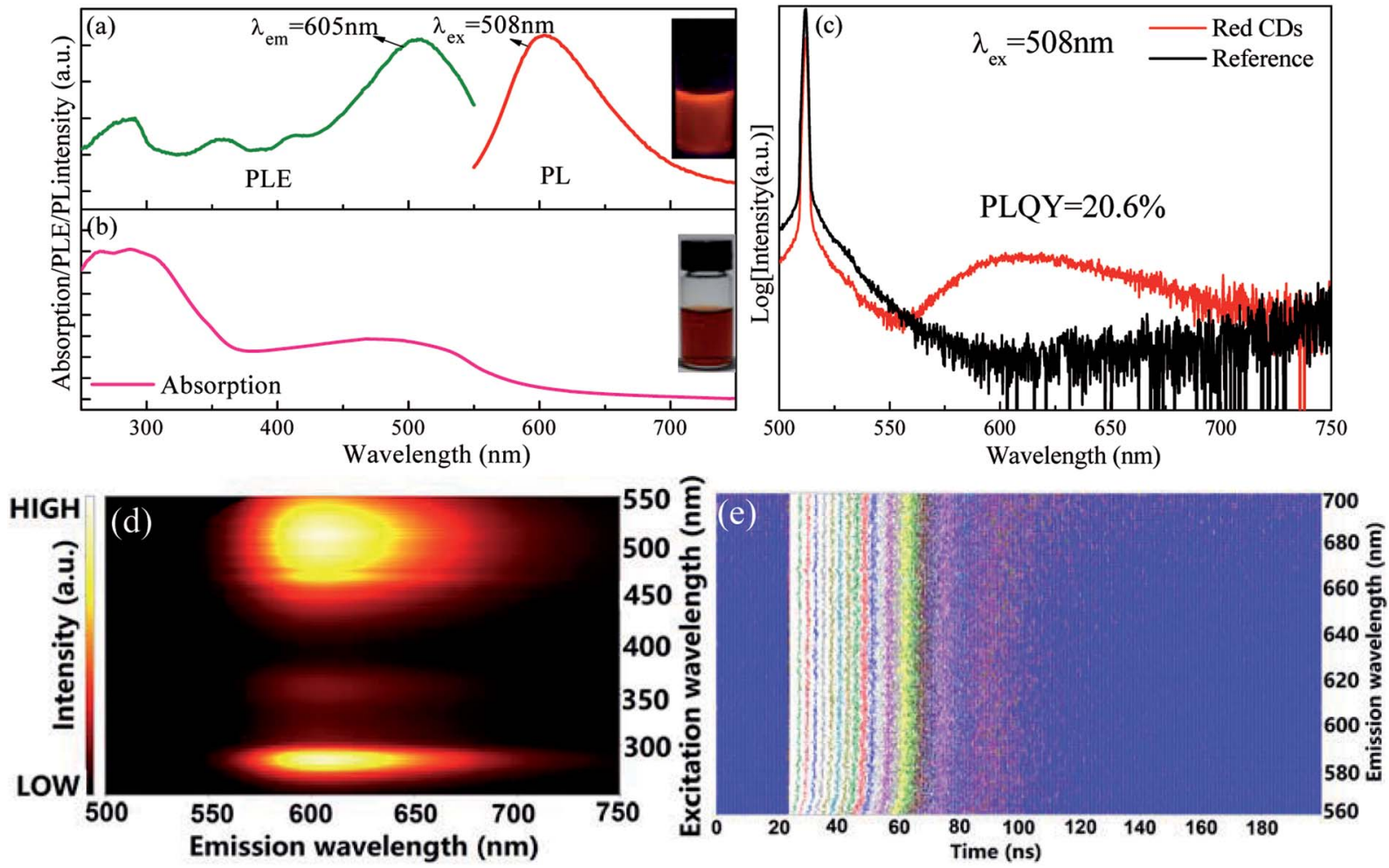

Fig. 1 Optical characterizations of the as-prepared red CDs. (a) PLE/PL spectra, (b) absorption spectrum, (c) excitation and emission spectra of CDs and reference to determine PLQY, (d) two-dimensional excitation-emission mapping and (e) contour plot of time-resolved data in the emission spectral range of 560-700 nm. Insets are photographs of CD solution under daylight (a) and UV (365 nm) lamp irradiation (b). 

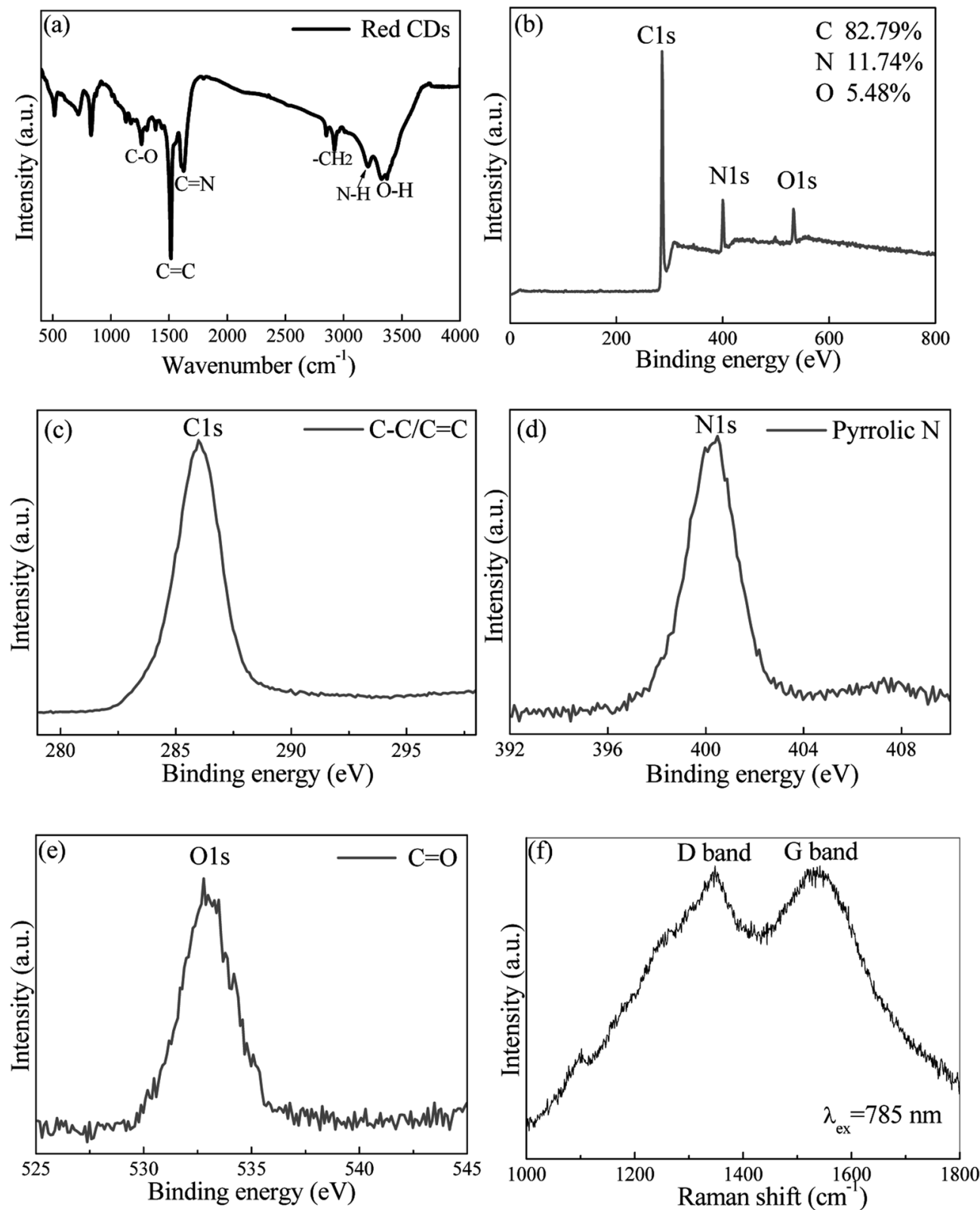

Fig. 2 (a) FTIR spectrum, (b) XPS full-survey and high-resolution XPS (c) C 1s, (d) N 1s and (e) O 1s spectra. (f) Raman spectrum of the as-prepared red $C D s$.

voltage. Absorption and emission spectra were recorded on an Edinburgh Instruments (EI) FS5 spectrofluorometer equipped with continuous $(150 \mathrm{~W})$ and pulsed xenon lamps. Excitationemission mappings of red CDs were recorded by continuously changing the excitation wavelength with a fixed step of $1 \mathrm{~nm}$, and the offset between excitation wavelength and emission was set at $30 \mathrm{~nm}$ to reduce scattering light. Absolute photoluminescence quantum yield (PLQY), defined as the ratio of emitted photons to absorbed ones, was determined by a spectrofluorometer (FS5) equipped with an integrating sphere. Time-resolved spectra of red CDs were performed on a fluorescent lifetime spectrometer (LifeSpec-II, EI) based on a timecorrelated single-photon-counting technique under the excitation of $475 \mathrm{~nm}$ picoseconds laser. The lifetimes were evaluated via the equation of $\tau=\int I(t) \mathrm{d} t / I_{0}$, where $I(t)$ is the time-related luminescence intensity and $I_{0}$ is the peak intensity. Electroluminescence (EL) spectra, Commission Internationale de L'Eclairage (CIE) chromaticity coordinates, color rendering index (CRI), correlated color temperature (CCT), and luminous efficiency (LE) of the constructed wLEDs were recorded in a HAAS-2000 spectroradiometer (Everfine) under an operating current of 10-200 mA. All the experiments were performed at room temperature.

\section{Results and discussion}

$p$-PD acting as carbon source and IPA as solvent was adopted to fabricated red CDs by a one-pot solvothermal reaction. Herein, 

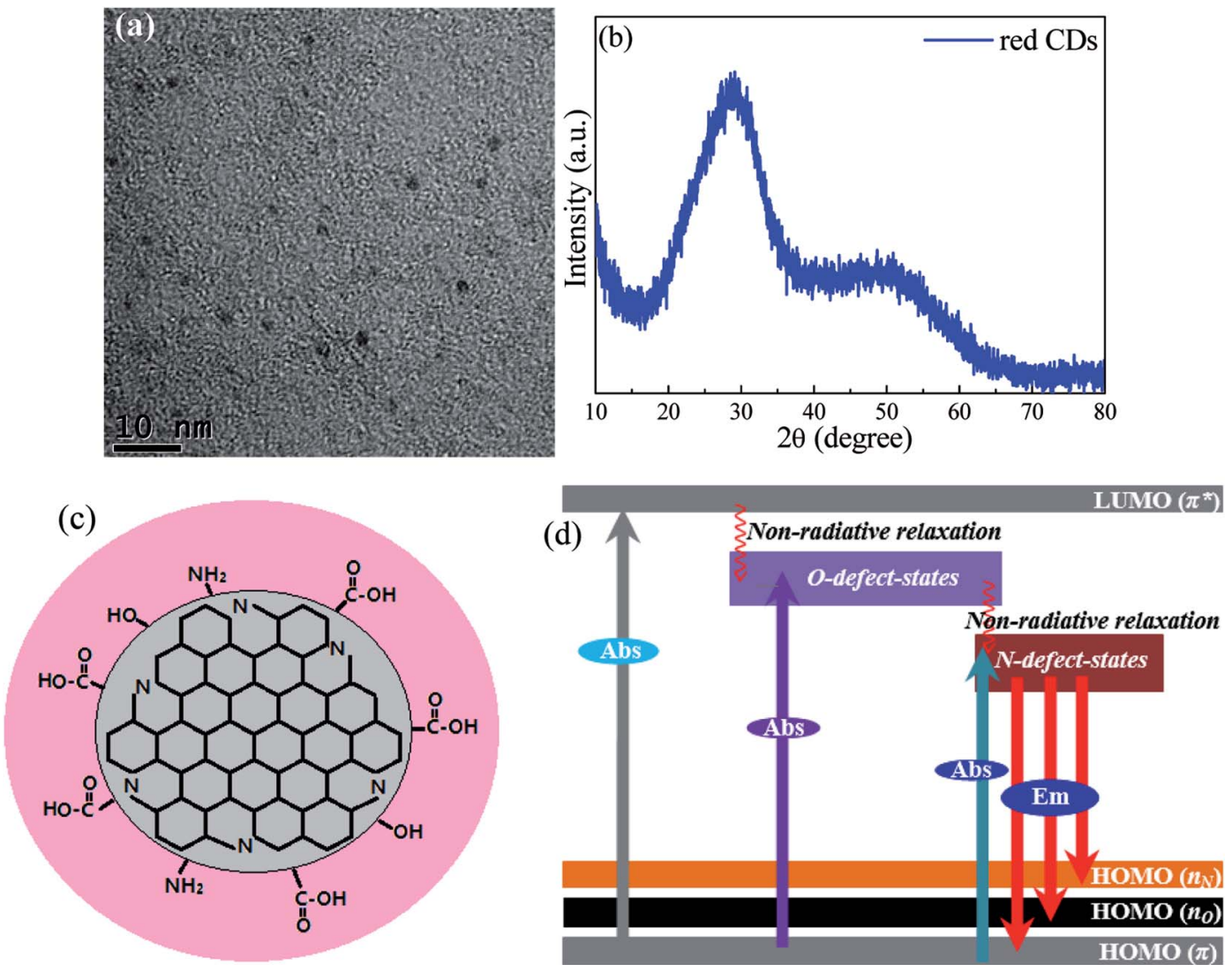

Fig. 3 (a) TEM image and (b) XRD pattern of red CDs. (c) Proposed structural model and (d) the corresponding absorption (Abs) and red emission $(\mathrm{Em})$ mechanisms for the investigated CDs.

the presence of $p$-PD was crucial for obtaining CDs with longwavelength emission as $p$-PD is the only carbon source. In fact, experimental conditions, including reaction temperature, time and $p$-PD content, significantly affect optical performance of the as-prepared CDs (Fig. S1 $\dagger$ ), and the optimal conditions for efficient luminescence of CDs are determined to be $200{ }^{\circ} \mathrm{C}$, $1 \mathrm{~h}$ and $0.25 \mathrm{mmol}$. As shown in Fig. 1a, a broad red emission band centered at $605 \mathrm{~nm}$ is detected in the PL spectrum and multiple excitation bands are observed in the corresponding PLE spectrum. A typical peak at $270 \mathrm{~nm}$ assigned to $\pi-\pi^{*}$ (aromatic $\mathrm{C}=\mathrm{C}$ ) transition and a broad absorption band in the wavelength region of 400-600 $\mathrm{nm}$ probably attributed to transitions from surface defect states to ground state are observed in the UV-visible absorption spectrum (Fig. 1b). As revealed in Fig. 1c, absolute PLQY of red CDs is determined to be as high as $20.6 \%$, being close to the values (4-25\%) for those previously reported orange or red CDs. ${ }^{17,26-28}$ Importantly, the present red CDs exhibit excitation-independent emission feature, i.e., upon the variation of excitation light wavelength, the emission intensity gradually changes but the emission peak position does not vary, as evidenced in Fig. 1d and S2a. $†$ This result indicates that the luminescence is probably originated from an individual emitting state in red CDs. Indeed, PL lifetime curves (Fig. S2b $\dagger$ ) and the corresponding contour plot (Fig. 1e) evidence that no obvious change in decay lifetime is found with increase of detecting emission wavelength of red CDs from $560 \mathrm{~nm}$ to $700 \mathrm{~nm}$. All these experimental results verify that only one type of emission state in the prepared CDs is dominantly responsible for the observed red luminescence.

The surface states and element analysis of red CDs were characterized by X-ray photoelectron spectroscopy (XPS), Fourier transform infrared (FTIR) and Raman spectra. For FTIR spectrum, amine $\left(3226 \mathrm{~cm}^{-1}\right)$, methylene $\left(2920 \mathrm{~cm}^{-1}\right), \mathrm{C}=\mathrm{C}$ (1491 $\left.\mathrm{cm}^{-1}\right), \mathrm{O}-\mathrm{H}\left(3425 \mathrm{~cm}^{-1}\right), \mathrm{C}=\mathrm{N}\left(1642 \mathrm{~cm}^{-1}\right)$ and $\mathrm{C}-\mathrm{O}$ $\left(1124 \mathrm{~cm}^{-1}\right)$ functional groups or chemical bonds are detected on the surface of red CDs (Fig. 2a). ${ }^{29,30}$ These FTIR assignments are further verified by XPS analysis. The red CDs mainly contain $\mathrm{C}, \mathrm{N}$ and $\mathrm{O}$ elements (Fig. 2b). High-resolution $\mathrm{C}$ 1s XPS spectrum exhibits a distinctive peak at $284.7 \mathrm{eV}$ assigned to $\mathrm{C}=\mathrm{C} / \mathrm{C}-$ $\mathrm{C}$ (Fig. 2c), N 1s XPS spectrum shows a characteristic peak at 400.5 attributed to pyrrolic-like $\mathrm{N}$ (Fig. 2d), and O 1s XPS spectrum reveals a peak of $\mathrm{C}=\mathrm{O}$ at $533.1 \mathrm{eV}$ (Fig. 2e). ${ }^{31,32}$ Additionally, Raman spectrum in the $1000-1800 \mathrm{~cm}^{-1}$ region (Fig. 2f) was measured under $785 \mathrm{~nm}$ laser excitation to characterize the intrinsic structure of red CDs, where $\mathrm{G}$ band centered at $1540 \mathrm{~cm}^{-1}$ attributes to graphitic $\mathrm{sp}^{2}$ carbon and D band located at $1350 \mathrm{~cm}^{-1}$ originates from defects on the surface of red CDs. ${ }^{33-35}$

TEM image, shown in Fig. 3a, demonstrates that the synthesized red CDs are monodispersed with sizes in the range of 3-5 nm. XRD pattern (Fig. 3b) shows two broad humps, confirming the amorphous structure of red CDs. Accordingly, based on the above structural and spectroscopic characterizations, a possible structural model of the present CDs and the 

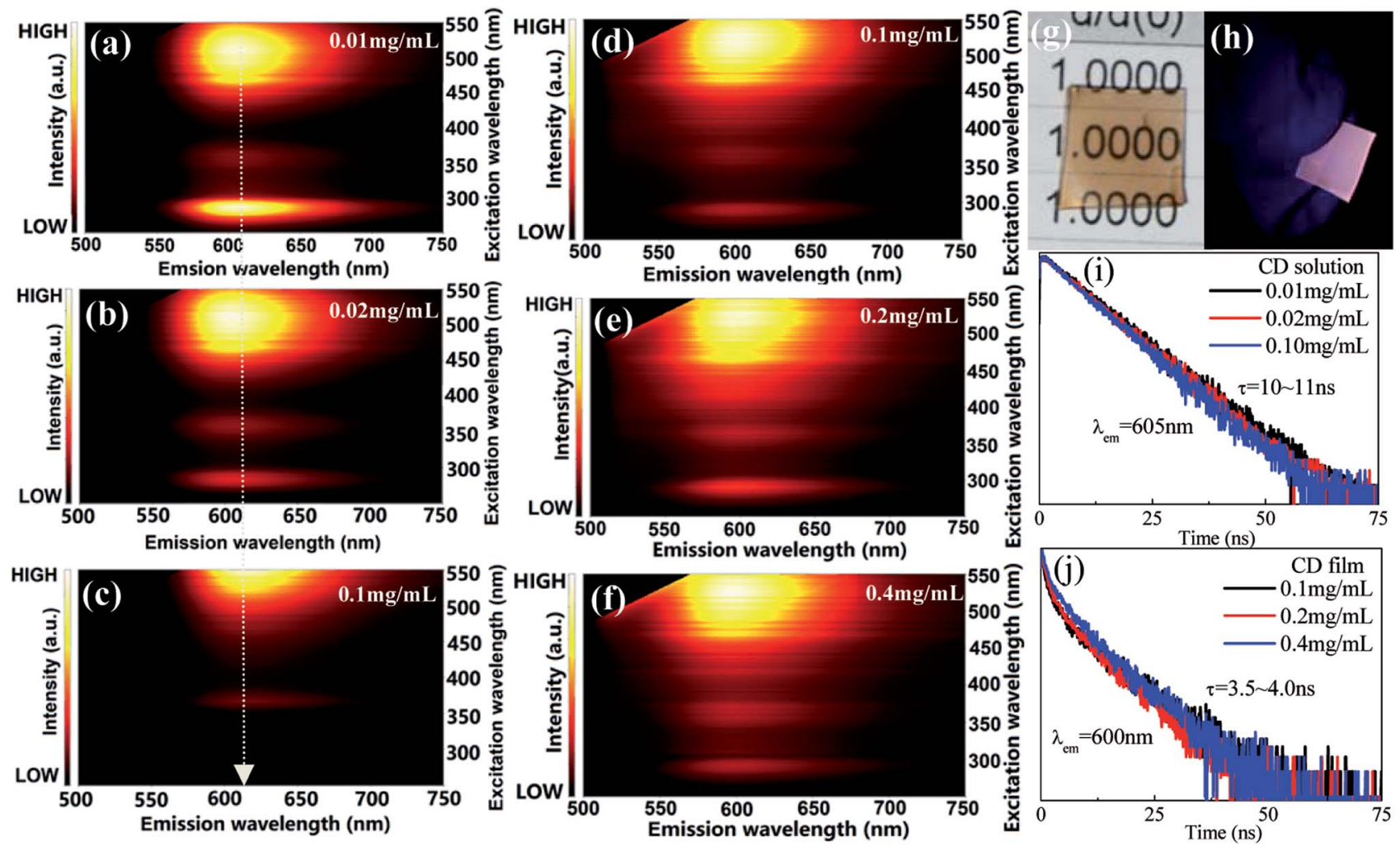

Fig. 4 Two-dimensional excitation-emission mappings of $(a-c)$ red CDs solutions (from top to bottom: the concentration of CDs gradually increases from 0.01 to 0.02 to $0.1 \mathrm{mg} \mathrm{mL}^{-1}$ ) and $(\mathrm{d}-\mathrm{f}$ ) red CDs films (from top to bottom: the concentration of CDs in PVA film increases from 0.1 to 0.2 to $0.4 \mathrm{mg} \mathrm{mL}^{-1}$ ). Photographs of red CDs in PVA film under irradiation of (g) sunlight and (h) blue light. Typical time-resolved decay curves of (i) red $C D$ solutions and (j) red CD films.

related energy-state structure responsible for multi-state absorption and red luminescence were proposed, as schematically illustrated in Fig. 3c and d. During solvothermal reaction, $p$-PD acting as both C source and $\mathrm{N}$ source could assemble into $\mathrm{N}$-doped CDs through the dehydrolysis, carbonization and nitridation processes accompanied with the formation of organic functional groups on the surfaces including $\mathrm{COOH}, \mathrm{C}=$ $\mathrm{O}, \mathrm{C}=\mathrm{N}, \mathrm{OH}$ and $\mathrm{NH}_{2}$ and the resulted $\mathrm{O}-/ \mathrm{N}$-related defect states (Fig. 3c). As proposed in Fig. 3d, the absorption/excitation bands in the UV region are originated from $\pi \rightarrow \pi^{*}$ (HOMO $\rightarrow$ LUMO) transition of $\mathrm{C}=\mathrm{C}$ bonds of $\mathrm{sp}^{2} \mathrm{C}$ domain, and those in the visible region are assigned to the transitions from $\pi$ states to $\mathrm{O}$ - and N-related defect states located within energy gap of CDs. As a result, upon the excitation of UV-visible light, the electrons in LUMO state and O-related defect states will non-radiatively relax into N-related defect states, from which red emission yields via radiative transition to HOMO states. To evidence surface defect-state induced red luminescence in the present CDs, $\mathrm{pH}$ dependent emission/absorption behaviors were investigated. For the PL spectra (Fig. S3a †), the emission peak position and emission intensity significantly vary when $\mathrm{pH}$ value deviates from 7; for the absorption spectra (Fig. S3b5†), HOMO $\rightarrow \operatorname{LUMO}\left(\pi \rightarrow \pi^{*}\right)$ transition of the graphitic C domain in the UV region shows no obvious change while the absorption transitions from $\pi$ ground state to $\mathrm{O}-\mathrm{N}$-related defect states exhibit remarkable $\mathrm{pH}$-dependent. All these results confirm that the red emission of CDs is actually caused by the N-related defect states, and the introduction of $\mathrm{H}^{+}$and $\mathrm{OH}^{-}$has a great influence on these surface emitting-states.

As schematically illustrated in Fig. $4 \mathrm{a}-\mathrm{c}$, the luminescence of red CD solutions with different CD concentrations were studied. With increase of $\mathrm{CD}$ content, the excitation peak position of red CDs gradually red-shifts towards long-wavelength, i.e., the optimal excitation wavelength required for efficient red emission changes from $510 \mathrm{~nm}$ to $550 \mathrm{~nm}$, which are actually due to reabsorption effect of high-content CDs in solution. To enable their application in solid-state-lighting, C-dot solid powders were further obtained by the vacuum freeze-drying method. Unfortunately, no any red luminescence was detected under the excitation of UV-visible light, probably attributing to the aggregation-induced emission quenching. To solve this issue, herein PVA was utilized as the supporting matrix to prepare red CD solid films with adjustable shape, size and thickness. Different to CD solution, CD solid films show invariable emissions with increase of CD content in PVA and no red-shift of excitation wavelength is found (Fig. $4 \mathrm{~d}-\mathrm{f}$ ). Importantly, the prepared CD films can be effectively excited by blue light, enabling their application in white light-emitting diode as red color converter. The prepared CD film shows high transparency under daylight (Fig. 4g) and yields bright red luminescence under the irradiation of blue light (Fig. 4h). Notably, compared to $\mathrm{CD}$ solution, the luminescence of CD solid film exhibits slight blue-shift from $605 \mathrm{~nm}$ to $600 \mathrm{~nm}$, probably attributing to the modification of defect-related energy states induced by 

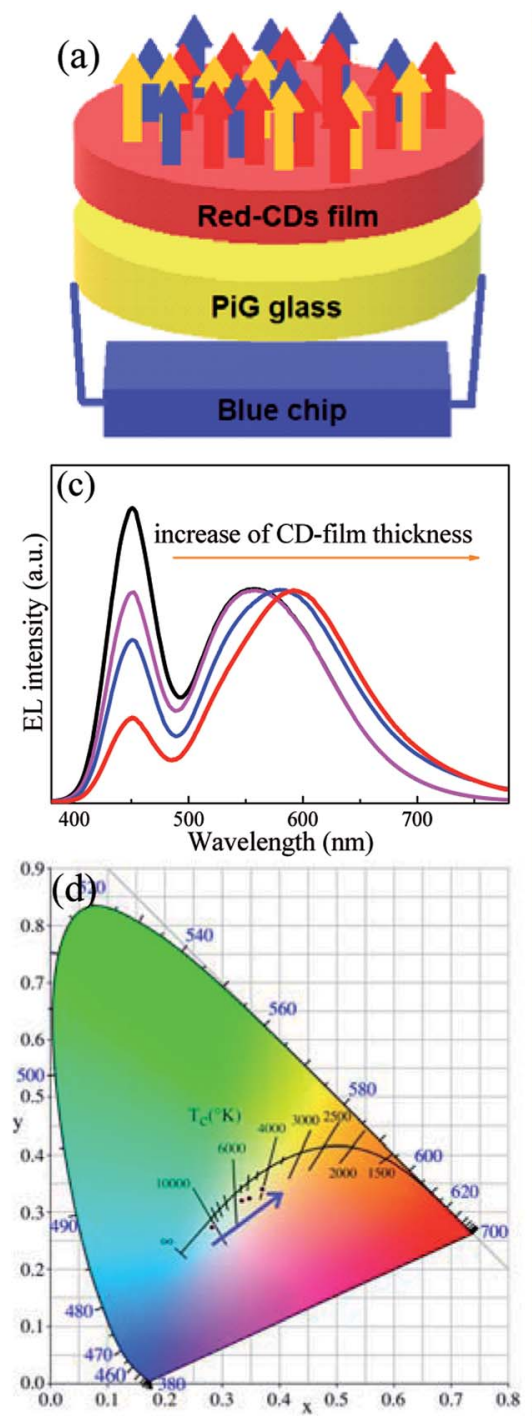
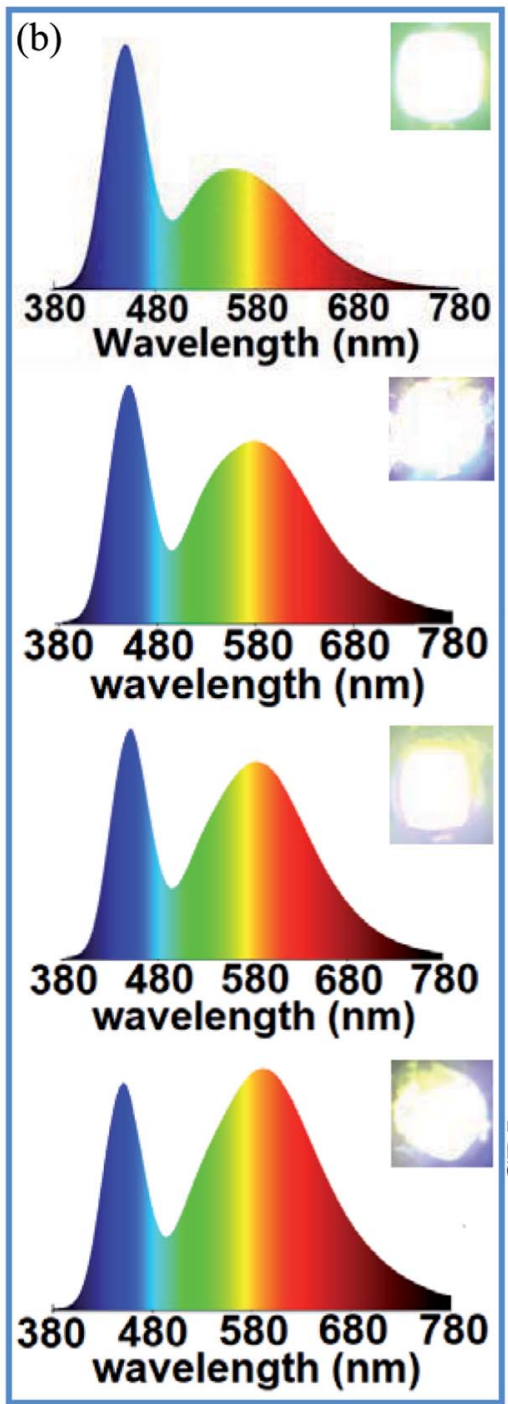
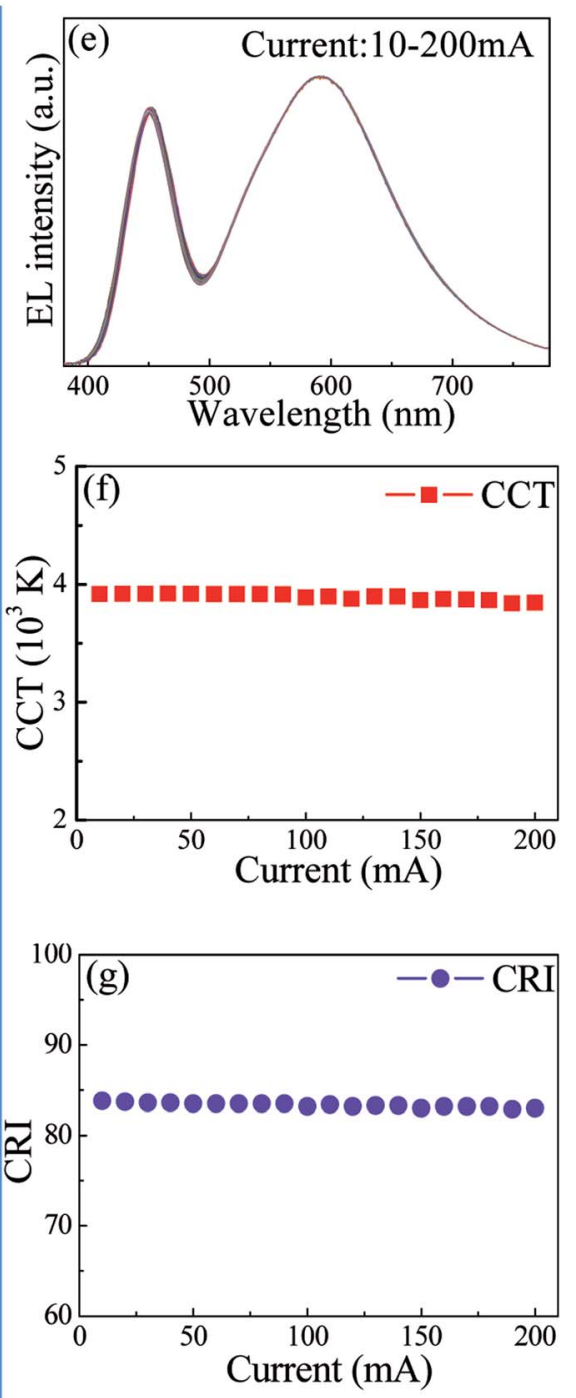

Fig. 5 (a) Schematic illustration of the structure of prototype LED device. (b) EL spectra of the constructed wLEDs by coupling commercial blue InGaN chip with yellow Ce:YAG PiG and red CD film as the color converters (film thickness increases from left to right: 0, 0.5, 1.0, 1.5 mm; denoted as S1, S2, S3 and S4, respectively). Insets are the corresponding WLEDs in operation (driven current: $100 \mathrm{~mA}$ ). Dependence of (c) normalized EL spectra and (d) CIE chromaticity coordinates of wLEDs on the thicknesses of CD film driven by $100 \mathrm{~mA}$ operating current. (e) EL spectra, (f) the corresponding CCT and (g) CRI for a typical wLED as a function of operating current (10-200 mA).

interaction between C-dots and PVA matrix. As shown in Fig. 4i and $\mathrm{g}$, the variation of $\mathrm{CD}$ contents will not result in significant change of decay lifetimes, however, the lifetime of CD solid film is obviously shorter than that of CD solution.

As a proof-of-concept experiment, wLED devices were constructed by coupling commercial InGaN blue chip with yellow Ce:YAG PiG and red CD film, as schematically illustrated in Fig. 5a. EL spectra of wLEDs driven by $100 \mathrm{~mA}$ operating current consist of blue emission attributed to InGaN chip and yellowred luminescence originated from Ce:YAG and CDs (Fig. 5b). With increase of thickness of CD film, the yellow-red emission relative to blue one gradually enhances and the emission wavelength shifts to red light region (Fig. 5c). As a result, the shifting of CIE color coordinates towards red region (Fig. $5 \mathrm{~d}$, Table 1), the decreasing of CCT from 10714 to 3995 (Table 1), the increasing of CRI from 75 to 85 (Table 1) are clearly observed. Correspondingly, the emitting color of wLED changes from cold white to warm white, as evidenced in insets of Fig. 5b. Finally, the influence of driving current on optoelectronic properties for a typical wLED was investigated. As revealed in Fig. 5e-g, no remarkable change of EL spectra, CCT and CRI is

Table 1 Optoelectronic parameters for the constructed wLEDs by coupling blue chip with yellow Ce:YAG PiG and red CD film with different thicknesses under the operation current of $100 \mathrm{~mA}$

\begin{tabular}{lllll}
\hline Device & $\begin{array}{l}\text { CD-film } \\
\text { thickness }(\mathrm{mm})\end{array}$ & Color coordinates & CCT & CRI \\
\hline S1 & 0 & $(0.283,0.274)$ & 10714 & 75 \\
S2 & 0.5 & $(0.335,0.319)$ & 5359 & 81 \\
S3 & 1.0 & $(0.348,0.324)$ & 4772 & 84 \\
S4 & 1.5 & $(0.371,0.341)$ & 3994 & 85
\end{tabular}


observed with increase of operating current form $10 \mathrm{~mA}$ to 200 $\mathrm{mA}$, proving that the developed CD solid film is applicable as red color converter in solid-state-lighting.

\section{Conclusions}

In summary, $\mathrm{N}$-doped carbon dots were synthesized via a facile solvothermal reaction using $p$-PD as $\mathrm{C} / \mathrm{N}$ source and IPA as solvent. The as-prepared carbon dots showed bright excitationinsensitive red luminescence, emission-independent decay and absolute PLQY of $20.6 \%$. Microstructural and optical spectroscopic characterizations confirmed that the observed red emission was originated from $\mathrm{N}$-related defect-state on the surfaces of carbon dots. Additionally, red emissive solid films were fabricated by incorporating carbon dots into PVA matrix. Benefited from intense blue light absorption and bright red luminescence, the as-prepared carbon solid films were demonstrated to have promising applications in solid-statelighting as red color converter.

\section{Conflicts of interest}

The authors declare no competing financial interest.

\section{Acknowledgements}

This research was supported by Zhejiang Provincial Natural Science Foundation of China (LR15E020001), National Natural Science Foundation of China (51572065), and 151 Talent's Projects in the Second Level of Zhejiang Province.

\section{References}

1 Z. F. Wang, F. L. Yuan, X. H. Li, Y. C. Li, H. Z. Zhong, L. Z. Fan and S. H. Yang, Adv. Mater., 2017, 29, 1702910.

2 X. H. Gao, C. Du, Z. H. Zhuang and W. Chen, J. Mater. Chem. C, 2016, 4, 6927-6945.

3 F. L. Yuan, Z. B. Wang, X. H. Li, Y. C. Li, Z. A. Tan, L. Z. Fan and S. H. Yang, Adv. Mater., 2017, 29, 1604436.

4 K. K. R. Datta, G. G. Qi, R. Zboril and E. P. Giannelis, J. Mater. Chem. C, 2016, 4, 9798-9803.

5 D. Q. Chen, H. B. Gao, X. Chen, G. L. Fang, S. Yuan and Y. J. Yuan, ACS Photonics, 2017, 4, 2352-2358.

6 H. Ali, S. K. Bhunia, C. Dalal and N. R. Jana, ACS Appl. Mater. Interfaces, 2016, 8, 9305-9313.

7 Y. B. Liu, L. Zhou, Y. N. Li, R. P. Deng and H. J. Zhang, Nanoscale, 2017, 9, 491-496.

8 M. I. J. Stich, M. Schaeferling and O. S. Wolfbeis, Adv. Mater., 2009, 21, 2216.

9 M. Vedamalai, A. P. Periasamy, C. W. Wang, Y. T. Tseng, L. C. Ho, C. C. Shih and H. T. Chang, Nanoscale, 2014, 6, 13119-13125.

10 J. Y. Zhu, X. Bai, Y. Zhai, X. Chen, Y. S. Zhu, G. C. Pan, H. Z. Zhang, B. A. Dong and H. W. Song, J. Mater. Chem. C, 2017, 5, 11416-11420.

11 T. L. Feng, Q. S. Zeng, S. Y. Lu, X. J. Yan, J. J. Liu, S. Y. Tao, M. X. Yang and B. Yang, ACS Photonics, 2018, 5, 502-510.
12 S. L. Hu, A. Trinchi, P. Atkin and I. Cole, Angew. Chem., Int. Ed., 2015, 54, 2970-2974.

13 C. X. Wang, H. H. Lin, Z. Z. Xu, Y. J. Huang, M. G. Humphrey and C. Zhang, ACS Appl. Mater. Interfaces, 2016, 8, 66216628.

14 W. Kown, S. Do, J. Lee, S. Hwang, J. K. Kim and S. W. Rhee, Chem. Mater., 2013, 25, 1893-1899.

15 H. Nie, M. J. Li, Q. S. Li, S. J. Liang, Y. Y. Tan, L. Sheng, W. Shi and S. X. A. Zhang, Chem. Mater., 2014, 10, 3104-3112.

16 S. J. Zhu, Q. N. Meng, L. Wang, J. H. Zhang, Y. B. Song, H. Jin, K. Zhang, H. C. Sun, H. Y. Wang and B. Yang, Angew. Chem., Int. Ed., 2013, 52, 3953-3957.

17 K. Hola, M. Sudolska, S. Kalytchuk, D. Nachtigallova, A. L. Rogach, M. Otyepka and R. Zboril, ACS Nano, 2017, 11, 12402-12410.

18 Y. H. Chen, M. T. Zheng, Y. Xiao, H. W. Dong, H. R. Zhang, J. L. Zhuang, H. Hu, B. F. Lei and Y. L. Liu, Adv. Mater., 2016, 28, 312-318.

19 D. Zhou, L. Di, P. T. Jing, Y. C. Zhai, D. H. Shen, S. N. Qu and A. L. Rogach, Chem. Mater., 2017, 29, 1779-1787.

20 P. J. G. Luo, F. Yang, S. T. Yang, S. K. SonKar, L. J. Yang, J. J. Broglie, Y. Liu and Y. P. Sun, RSC Adv., 2014, 4, 1079110807.

21 S. Sun, L. Zhang, K. Jiang, A. G. Wu and H. W. Lin, Chem. Mater., 2016, 28, 8659-8668.

22 H. Wang, C. Sun, X. R. Chen, Y. Zhang, V. L. Colvin, Q. Rice, J. Seo, S. Y. Feng, S. N. Wang and W. W. Yu, Nanoscale, 2017, 9, 1909-1915.

23 D. Q. Chen, W. W. Wei, Y. J. Yuan, Y. Yang, Z. Y. Wan and P. Huang, J. Mater. Chem. C, 2016, 4, 9027-9035.

24 H. Ding, S. B. Yu, J. S. Wei and H. M. Xiong, ACS Nano, 2016, 10, 484-491.

25 K. Jiang, S. Sun, L. Zhang, Y. Lu, A. G. Wu, C. Z. Cai and H. W. Lin, Angew. Chem., Int. Ed., 2015, 54, 5360-5363.

26 Y. Zhai, Y. Wang, D. Li, D. Zhou, P. Jing, D. Shen and S. Qu, J. Colloid Interface Sci., 2018, 528, 281-288.

27 C. Li, Y. Wang, X. Zhang, X. Guo, X. Kang, L. Du and Y. Liu, J. Colloid Interface Sci., 2018, 526, 487-496.

28 Y. Y. Din, X. J. Gong, Y. Liu, W. J. Lu, Y. F. Gao, M. Xian, S. M. Huang and C. Dong, Talanta, 2018, 189, 8-15.

29 C. H. Yang, S. J. Zhu, Z. L. Li, Z. Li, C. Chen, L. Sun, W. Tang, R. Liu, Y. Sun and M. Yu, Chem. Commun., 2016, 52, 111912111914.

30 H. Ding, J. S. Wei and H. M. Xiong, Nanoscale, 2014, 6, 13817-13823.

31 S. N. Baker and G. A. Baker, Angew. Chem., Int. Ed., 2010, 49, 6726-6744.

32 H. T. Li, X. D. He, Z. H. Kang, H. Huang, Y. Liu, J. Liu, J. L. Liu, S. Y. Lian, C. H. A. Tsang, X. B. Yang and S. T. Lee, Angew. Chem., Int. Ed., 2010, 49, 4430-4434.

33 Y. X. Fang, S. J. Guo, D. Li, C. Z. Zhu, W. Ren, S. J. Dong and E. K. Wang, ACS Nano, 2012, 6, 400-409.

34 C. Zhu, J. Zhai and S. Dong, Chem. Commun., 2012, 48, 93679369.

35 S. Zhu, J. Zhang and S. Tang, Adv. Funct. Mater., 2012, 22, 4732-4740. 